\title{
Performativité de l'écrit et travail de maintenance
}

\author{
Jérôme DENIS \\ LTCI (UMR5141) CNRS - Telecom ParisTech \\ Département Sciences Économiques et Sociales \\ jerome.denis@telecom-paristech.fr \\ David PONTILLE \\ IIAC - CNRS (UMR 8177) / EHESS (LC 12) \\ Équipe «Anthropologie de l'écriture » \\ pontille@ehess.fr
}

\section{Résumé}

L'analyse sociologique des objets graphiques qui ordonnent l'environnement se concentre généralement sur les ajustements effectués pour les constituer en ressources pour l'action, faisant des usages le principal ressort de leur performativité. À partir du cas de la signalétique du métro, cet article renverse cette perspective et se focalise sur l'activité d'aménagement des lieux. II cherche ainsi à éviter de considérer les artefacts graphiques comme des objets inertes, et de faire de l'immuabilité une propriété intrinsèque de l'écrit. II montre au contraire l'importance cruciale du travail de maintenance qui entretient au jour le jour les qualités spatiales et matérielles des objets et leur assure une force performative sans cesse réactualisée.

Denis, J. et Pontille, D. 2010. Performativité de l'écrit et travail de maintenance Réseaux, 163, p. 105-130. 
Avec les feux tricolores, les bornes, les marquages au sol, les potelets ou les trottoirs, les panneaux de la signalétique font partie des innombrables artefacts qui organisent le monde et la circulation des entités qui le traversent. Parce qu'ils sont directement disponibles dans l'environnement, ces objets tiennent une place particulière dans nos actions ordinaires, au même titre que ceux qu'ont étudié les auteurs de l'anthropologie cognitive et de " l'action située »(Hutchins, 1995 ; Lave, 1988 ; Suchman, 1987). Ces derniers ont notamment montré que nous engageons des dialogues réguliers avec les artefacts, négociant plus ou moins explicitement leur statut de ressources ou de contraintes au fil de l'activité.

Parmi eux, J. Lave (1988) a proposé une conceptualisation originale de la manière dont les personnes interagissent avec leur environnement matériel et informationnel, en partant du cas des clients de supermarché. Pour rendre compte de ce processus, elle utilise les notions de settings et d'arenas. La distinction entre les deux lui permet d'insister sur le fait que le cadre pertinent de l'action (le setting) est le résultat provisoire d'un ajustement dynamique et situé, effectué à partir d'une recomposition des éléments matériels présents dans l'environnement (l'arena). Cette perspective est féconde pour comprendre le caractère endogène de ce qui peut être considéré comme le contexte de l'activité. Elle demeure cependant largement asymétrique. Alors que, dans ces analyses, les settings sont riches et faits d'ajustements complexes, les arenas sont dépouillées et considérées comme un préalable à l'action : avant l'intervention des personnes qui les mobilisent avec plus ou moins de difficulté pour produire des settings, les espaces et leurs artefacts sont inertes, reflets directs d'enjeux politiques qui les dépassent ${ }^{1}$.

Ce type d'approche est très fréquent à propos de la signalétique. II s'est traduit dans des recherches qui ont essentiellement identifié la complexité et l'inventivité de ses usages en documentant systématiquement le travail local nécessaire à l'accomplissement d'une activité orientée par des panneaux directionnels (Sharrock et Anderson, 1979 ; Timpf, 2002). La focalisation sur les usages a aussi permis de montrer que de tels dispositifs ne suffisaient pas à la réussite des parcours et que leur usage consistait en grande partie à leur articulation, en situation, à d'autres ressources (Lévy, 2001).

Or, pour interroger la force performative d'artefacts comme ceux qui composent un système signalétique, le centrage sur les usages s'avère insuffisant. Surtout s'il amène à considérer que les artefacts et l'espace qu'ils équipent sont des « données » qui ne sont considérées comme dynamiques qu'à partir de la seule observation de leur mobilisation par des usagers. Les environnements de l'action, d'autant plus lorsqu'il s'agit de lieux publics, sont des espaces organisés. Ils font l'objet d'activités dédiées à leur mise en ordre (Relieu, 1999 ; Relieu et Terzi, 2004). La présence même d'artefacts dans l'espace résulte d'un travail qu'il est essentiel d'étudier en profondeur afin d'en comprendre la force performative. Pour reprendre les termes de J. Lave, nous proposons donc, à partir du cas de la signalétique du métro parisien, d'analyser le travail d'aménagement des arenas. Notre objectif n'est pas ici d'entrer dans le détail ethnographique de notre enquête que nous avons exposée ailleurs (Denis et Pontille 2010a, 2010b), mais de chercher à montrer ce qu'une telle perspective, notamment parce qu'elle permet de souligner le rôle des activités de maintenance, apporte à la compréhension de la performativité des artefacts.

Par ailleurs, en temps qu'objet de recherche, la signalétique ouvre une perspective d'analyse supplémentaire. Porteurs d'inscriptions, de mots et d'icônes, les panneaux qui la composent sont des artefacts hybrides. Leurs caractéristiques graphiques et langagières comptent autant que leur matérialité et leur position dans l'environnement. Ils relèvent de ce que $A$.

\footnotetext{
1 « The supermarket as arena is the product of patterns of capital formation and political economy (...) providing a
} higher-order institutional frame-work within which setting is constituted » (Lave, 1988 p. 151). 
Petrucci (1993) appelle des « écritures exposées ». Interroger ce type d'objets nous permet donc également de poursuivre un programme de recherche consacré à la performativité de l'écrit, que ce soit dans les métiers juridiques (Fraenkel, 2006 ; Pontille, 2006, 2009), les activités bancaires (Denis, 2009a), la gestion des réseaux informatiques (Denis, 2009b), ou dans les lieux publics (Fraenkel, 2002, 2007 ; Artières et Rodak, 2008 ; Denis et Pontille, 2010a, 2010c). Notre focalisation sur le travail d'aménagement de la signalétique a ainsi pour but d'élargir l'analyse des écrits aux conditions de leur fabrication et de leur mise en forme, c'est-à-dire aux activités qui contribuent en coulisses à leur performativité.

Une telle approche représente à nos yeux un moyen de dépasser une aporie trop fréquente dans l'analyse de l'écrit. La tentation est grande, en effet, de rabattre la force de l'écrit sur des caractéristiques mises en évidence par des historiens pour des périodes précises (Eisenstein, 1991) ou des anthropologues dans des contextes particuliers (Goody, 1979). Les travaux qui adoptent cette posture font de la fixité, de la fiabilité, de la pérennité ou de la représentativité des propriétés intrinsèques qui suffisent à expliquer l'action des écrits. $\mathrm{Ce}$ qui revient finalement à dire que si les écrits sont performatifs, c'est avant tout parce qu'ils sont... des écrits. Faire de ces qualités des principes explicatifs consiste à basculer dans ce que M. Grosjean et M. Lacoste (1998) ont appelé les « illusions » de l'écrit et que l'on trouve au sein de certaines organisations où la transparence, la stabilité et la durabilité sont considérées comme des propriétés scripturales intrinsèques. Nous montrerons que, face à la variété des gestes que de multiples acteurs répètent quotidiennement pour garantir l'existence même des panneaux de la signalétique, de tels postulats sur l'écrit et sa performativité ne résistent pas. Loin d'être des qualités des artefacts graphiques en tant que tels, la fixité ou la pérennité sont les résultats fragiles et provisoires d'un travail délicat qui doit être continuellement recommencé.

Pour la signalétique, deux caractéristiques apparaissent particulièrement sensibles : l'immuabilité des inscriptions et la spatialité des énoncés. Elles ont fait l'objet de nombreuses réflexions théoriques sur lesquelles nous proposons de revenir brièvement.

\section{Performativité et stabilité des inscriptions}

La théorie de l'acteur-réseau est particulièrement utile pour explorer les conditions de la performativité des artefacts graphiques. Bien qu'on lui attribue volontiers le seul mouvement de réhabilitation des non-humains dans la théorie sociale, on néglige en effet trop souvent que les inscriptions y tiennent aussi une place cruciale. Dès La Vie de Laboratoire (Latour et Woolgar, 1988), l'accent est mis sur l'importance des actions consistant à coder, marquer, lire et écrire. La valeur des inscriptions produites par les divers dispositifs techniques est rapportée à la configuration du laboratoire scientifique où sont agencés des chercheurs, des appareils et des machines, des secrétaires, du matériel, de la littérature spécialisée, des techniciens. Les auteurs montrent que cet agencement sociotechnique rend les chercheurs capables de faire le tri entre les traces significatives et le bruit de fond qui émanent des instruments du laboratoire.

L'action des différentes inscriptions est rapportée à un processus d'associations généralisé par lequel les énoncés des articles scientifiques sont peu à peu chargés en matière et ainsi stabilisés (Callon, Law et Rip 1986). C'est par exemple le rôle attribué au Topofil Chaix (Latour, 1993) dans l'analyse des sols qui - combiné à d'autres instruments tels les pancartes numérotées quadrillant une partie de la forêt de Boa Vista, les étagères servant à classer et nommer des spécimens de plantes, et le pédocomparateur des différentes mottes de terre - permet aux scientifiques d'assurer progressivement la correspondance entre le monde (l'état d'un sol) et des signes (les inscriptions qui sont chargées de le représenter).

Les processus consistant à charger matériellement des énoncés ne se réduisent évidemment pas à l'activité scientifique. De nombreux cas, analysés à partir de la théorie de l'acteur-réseau, concernent plus largement la vie quotidienne (Bijker et Law, 1992). Et B. 
Latour et E. Hermant (1998) ont même documenté cette question à propos de certains énoncés présents dans l'espace urbain. Au cours de leur exploration des coulisses de Paris, ils ont montré comment des activités telles que la désignation des rues et la pose des plaques sont prises dans un vaste réseau où s'articulent un nombre considérable de traces écrites et de services administratifs : des dossiers, des chemises cartonnées, des formulaires, des bordereaux, des plans qui émanent du service de la Voirie, du service du Parcellaire, du service technique de la Documentation foncière, du service de la Nomenclature... Le succès de la désignation des rues est ici appréhendé comme le résultat de l'alignement de ces diverses inscriptions qui, fermement attachées les unes aux autres, donnent mutuellement consistance à chaque nom de rue.

Du point de vue de la théorie de l'acteur-réseau, l'écriture est ainsi envisagée de façon relationnelle. La force d'un énoncé ne réside jamais dans une inscription en tant que telle. Au même titre que d'autres entités, les artefacts écrits prennent non seulement leur signification à partir de la relation qu'ils entretiennent avec les autres, mais le caractère achevé de leur forme est lui-même une conséquence directe de cette relation. Dans cette conception sémiotique, chaque élément du réseau tient une place spécifique et agit fonctionnellement par rapport aux autres. Ce sont les positions syntaxiques relatives des entités qui contribuent à l'action du réseau dans son ensemble.

Par ailleurs, dans la théorie de l'acteur-réseau, la performativité des artefacts, qu'ils soient graphiques ou non, repose sur une autre caractéristique. Nous venons de le voir, elle est d'abord liée à la stabilité des relations qu'ils entretiennent au sein d'un réseau. Mais cette stabilité est elle-même appréhendée comme la condition de leur circulation. Pour B. Latour la force des artefacts est conditionnée à leur capacité à devenir des « mobiles immuables " (Latour, 1985), c'est-à-dire des objets qui peuvent circuler d'un point à un autre du réseau sans changer d'état, ni perdre leur forme.

La signalétique du métro parisien est un bon exemple pour comprendre comment des écrits exposés peuvent faire l'objet d'un tel processus de stabilisation. Au début des années 1990, une équipe de personnes d'horizons variés (cartographes, architectes, designer...) s'est rassemblée et a mis en avant auprès de la direction de la RATP ce qu'elle présentait comme un constat accablant : les couloirs du métro étaient peuplés de panneaux hétérogènes, changeants d'une station à l'autre. II fallait à leurs yeux mettre un terme à cette variation extrême, considérée comme un facteur de désordre. Ce constat a été entendu et l'équipe a finalement été chargée de réorganiser en profondeur - ou plutôt d'organiser tout court, de leur point de vue - l'ensemble de la signalétique (Denis et Pontille, 2010a). Deux aspects de cette rénovation sont particulièrement importants.

D'une part, la signalétique du métro est devenue, pour la première fois, l'objet d'une normalisation détaillée dont l'envergure est à la dimension des ambitions de ses instigateurs. Pour mettre un terme à la variabilité des panneaux, toutes leurs dimensions sont identifiées et arrêtées une fois pour toutes. Une série de modules est produite, qui vise à répondre à toutes les situations possibles dans les espaces dédiés aux voyageurs. Pour chaque type de panneaux, les règles de construction sont définies de façon exhaustive : dimensions, matières, et formes des supports, couleurs et disposition des inscriptions. II en est de même pour le vocabulaire, les appellations, l'orthographe et les abréviations à utiliser pour différents éléments (les noms de stations, de direction, de sortie, les correspondances, et les messages variés d'information). Avec une telle structuration des formes et la fixation de chaque composant au millimètre près, les éléments qui composent la signalétique sont désormais stabilisés et standardisés. Quelle que soit la variété des situations, l'instabilité des éléments graphiques est dorénavant contenue. À partir de 1996, chaque panneau de signalétique est devenu un objet standard et immuable.

D'autre part, cette rénovation s'est fondée sur la volonté d'organiser tous les panneaux en système : c'est ce que revendique le terme même de "signalétique ". La stabilité assurée 
par la normalisation est mise au service d'une interdépendance des modules graphiques. Ceux-ci doivent non seulement se répéter à l'identique, mais les éléments dont ils sont le support doivent eux-mêmes se retrouver d'un panneau à l'autre. Par exemple, la couleur spécifique affectée à chaque ligne de métro doit être strictement identique quelle que soit le support. De même, sur l'ensemble des panneaux, l'écriture en bleu foncé sur fond blanc indique les directions de lignes tandis que les lettres blanches sur fond bleu désignent les noms de stations et les sorties. L'immuabilité des panneaux tire ainsi toute sa signification de la mise en cohérence des rapports que chaque élément entretient avec les autres. Avec la normalisation, la signalétique est organisée comme un véritable dispositif graphique dans lequel des entités stabilisées occupent des positions relationnelles et assurent une fonction spécifique constante.

Pour les tenants de la théorie de l'acteur réseau, nous l'avons vu, l'immuabilité des entités n'assure le succès du réseau que si celles-ci sont corrélativement mobiles. C'est le cas des éléments de la signalétique : le document volumineux qui compose la charte graphique est non seulement présent dans les bureaux du département de normalisation, mais aussi dans l'atelier de fabrication des panneaux qui est rattaché au département de la maintenance. Les principes qui fondent l'immuabilité des panneaux, les règles qui fixent leurs propriétés, circulent entre différents sites, rendant ainsi parfaitement identiques, d'un endroit à l'autre, les entités précisément désignées comme une «flèche », un « jalonnement », un « panneau lumineux transversal », ou encore un «plan de trappe ». Les panneaux eux-mêmes, une fois produits, circulent également : ils sont transportés du lieu de leur fabrication jusqu'aux ateliers où ils sont réceptionnés et stockés, puis vers la station de métro dans laquelle ils doivent être posés.

Mais, dans le cas de la signalétique, c'est moins le fait que les éléments graphiques puissent être mobiles qui compte que leur capacité, in fine, à se fixer et à rester durablement en place. Les modules de la signalétique n'équipent véritablement l'espace des stations de métro qu'à partir du moment où ils deviennent des immobiles immuables, c'est-à-dire seulement lorsqu'ils sont installées à un emplacement précis et qu'ils y demeurent. Leur capacité à faire faire est suspendue à cet impératif. Ce point souligne ce qui semble être une spécificité des écrits exposés : leur performativité est étroitement liée au rapport particulier qu'ils entretiennent à l'espace.

\section{Écrits exposés et spatialité}

Deux séries de travaux sont particulièrement utiles pour comprendre l'importance de l'espace dans l'analyse de la performativité des écrits exposés.

R. Scollon et S.W. Scollon (2003) ont développé un programme de « géosémiotique » dans lequel ils s'attellent directement à cette question. Ils proposent de rompre avec la sémiologie traditionnelle qui restreint l'analyse à l'interprétation des signes " eux-mêmes » et plaident pour une étude des signes " en place ». Ils soulignent ainsi l'importance qu'il faut accorder à l'indexicalité, à partir d'une réflexion sur l'emplacement que les signes occupent dans le monde. L'exemple d'un panneau indiquant une issue de secours en cas d'incendie illustre bien ce problème (Scollon et Scollon, 2003, p. 28-31). La flèche montre dans quelle direction courir, et l'icône symbolisant une personne qui s'échappe des flammes est elle-même indexicale puisqu'elle court dans le même sens que la flèche. Mais un panneau de ce type ne vaut pas grand chose lorsqu'il est chez le fabricant ou dans les mains de celui qui va le poser. Ce n'est qu'une fois installé à un emplacement précis qu'il pourra véhiculer du sens. Dans ce modèle, le signe tire donc sa force et sa signification de propriétés spatiales. C'est parce qu'il partage un espace avec ce qu'il désigne qu'il peut prétendre à la performativité.

Dans son projet de théorie des actes d'écriture, B. Fraenkel $(2006,2007)$ propose elle aussi, dans une perspective sensiblement différente, de mettre l'espace au centre de l'analyse de la performativité de l'écrit. Pour se faire, elle part d'une discussion approfondie avec les 
travaux de J.-L. Austin (1970) qui abandonne lui-même la notion de performativité à l'issue de la septième conférence de Quand dire, c'est faire. Elle explique que le projet d'Austin, qui consistait à distinguer les énoncés constatifs des énoncés performatifs, est une impasse précisément parce qu'il néglige les qualités des énoncés écrits en ramenant l'énonciation à un ici et maintenant trop pauvre.

Fraenkel (2006) montre ainsi que lorsque Austin mobilise l'exemple du panneau " chien méchant ", il l'appréhende comme un énoncé oral et en fait un avertissement identique à celui que donnerait une personne présente sur les lieux. De la sorte, il semble passer complètement à côté des conditions de sa performativité. Premièrement, Fraenkel rappelle que le panneau n'est pas un énoncé qui flotte dans les airs en attente d'un énonciateur : il est installé quelque part. Son emplacement est totalement indissociable de sa dimension performative. Deuxièmement, le panneau est un objet. Sa forme et sa matière sont des qualités essentielles à sa capacité d'action. Ces deux éléments prolongent la réflexion proposée par les Scollon : ils soulignent le caractère situé de la performativité de l'écrit.

Mais, Fraenkel insiste sur un troisième point. C'est en accrochant le panneau " chien méchant » au portail d'une maison que celle-ci devient un lieu protégé. De même, c'est par l'installation d'un panneau à proximité d'un virage que celui-ci est transformé en virage dangereux. Dans cette perspective, la performativité des écrits tient aussi dans l'acte de mise en place : le site est modifié par l'adjonction d'un écrit dont la matérialité du support et l'emplacement sont cruciaux. Pour le dire autrement, dans ces cas, l'écrit fait l'espace, au même titre que pour G. Simmel ([1909] 1994) le pont fait les rives.

Ces travaux qui identifient les relations entre objets graphiques et espace permettent de spécifier la performativité de la signalétique du métro parisien. Le processus de normalisation dont cette dernière a été l'objet n'a en effet pas seulement engagé l'immuabilité des panneaux : il a concerné très directement leur emplacement. Chaque panneau du système s'est vu attribué une place singulière dans l'ensemble des stations.

Le déploiement d'une série de panneaux standardisés et ordonnés les uns par rapport aux autres a donc considérablement transformé l'espace de transport. L'environnement mis à la disposition des voyageurs s'est trouvé stabilisé sous une forme inédite. La standardisation des éléments graphiques a produit un assemblage particulier dans lequel " la distinction entre le bâtiment et ses signes, entre le texte et le territoire, devient floue " (Fuller, 2002, p. 236). Dans chaque station, les modules standardisés de la signalétique équipent désormais l'espace selon des modalités identiques. Quel que soit le point du réseau de transport par lequel on entre, les caractéristiques de l'environnement sont similaires.

Outre l'organisation d'une similarité généralisée, la répétition d'éléments standardisés dans les stations produit également des différences. Tout en stabilisant l'environnement, la signalétique opère un découpage de l'espace en régions. Équipé de modules graphiques spécifiques, le métro parisien se distingue très nettement des autres métros, tout comme il se détache du réseau ferroviaire de la SNCF avec lequel il partage pourtant certains espaces $^{2}$. À l'échelle du seul métro parisien, la stabilisation et l'ordonnancement de l'environnement se traduit aussi par une stricte division des lieux, qui va bien au-delà de la répartition des stations dans l'espace urbain. Avec la signalétique, chaque station se trouve divisée en zones : des halls d'entrée avec un plan du réseau et un plan de quartier ; des couloirs avec des flèches, des numéros de lignes et des noms de directions; des quais avec des plans du réseau, des correspondances, des noms de stations et des noms de sorties. Cette différenciation accomplit un ordonnancement à l'échelle du réseau. Les zones

2 Ces sites partagés font d'ailleurs l'objet d'importants débats pour garantir aux voyageurs un passage en douceur d'un système de transport à l'autre malgré le changement de système signalétique. Ils illustrent bien comment un même site est travaillé matériellement par des productions territoriales simultanées (Kärrholm, 2007). 
distinctes se répètent de stations en stations, et produisent, malgré les disparités architecturales, une série de lieux types.

Enfin, la mise en place d'une signalétique standardisée et ordonnée contribue à la transformation des voyageurs eux-mêmes. À l'occasion de son renouvellement, la signalétique est conçue comme une composante à part entière du service de transport. Elle est érigée au rang d'un véritable opérateur de mobilité, censé assurer aux usagers une circulation sans difficultés d'un point à un autre du réseau. L'immuabilité et l'immobilité des panneaux font donc des voyageurs des entités dont les déplacements doivent être le plus fluide possible. En ce sens, la signalétique est un cas paradigmatique des infrastructures immobiles de la mobilité qui assurent des appuis stables et durables à la circulation massive des personnes et des biens (Amin et Thrift, 2002 ; Lash et Urry, 1994).

Mais la fluidification opérée par la signalétique ne tient pas seulement à la stabilité des panneaux. Elle suppose d'attribuer aux voyageurs eux-mêmes une nouvelle fonction dans le réseau des entités qui composent les espaces de transport. Le renouvellement de la signalétique a installé dans les stations du métro une véritable politique de l'attention : les usagers y sont désormais configurés comme des êtres tout entier tournés vers l'environnement, attentifs aux multiples éléments graphiques censés garantir le succès de leurs déplacements (Denis et Pontille, 2010a, chap. 3).

L'ordonnancement opéré par la signalétique est donc multiple. La mise en place d'un système de modules graphiques standardisés transforme à la fois les propriétés de l'environnement et les qualités des voyageurs. Mais si la description de cette double transformation souligne l'ampleur et l'ambition « sur le papier » de la signalétique parisienne, elle laisse dans l'ombre une dimension essentielle à son accomplissement. La stabilisation des modules par la normalisation et la standardisation ne sont qu'un versant du processus qui consiste à charger les énoncés destinés à encadrer les déplacements dans le métro. Pour qu'un dispositif comme la signalétique existe effectivement dans les stations, pour que les flèches, les icônes, les noms des destinations et autres numéros de lignes soient durablement présents dans les espaces dédiés aux voyageurs, il doit faire l'objet d'un travail supplémentaire. La performativité d'une signalétique, aussi ambitieuse soit-elle, en dépend. C'est ce travail que nous proposons d'explorer maintenant. Ce pas supplémentaire nous permettra d'insister sur le fait que pour fabriquer un objet stable et durable dans un espace, il est souvent nécessaire d'effectuer certaines actions dans un autre (Law, 2002).

\section{Les multiples ressorts de la stabilité}

Dès lors que l'on entre dans ses coulisses, c'est-à-dire dans les locaux professionnels de l'entreprise de transport, la signalétique n'apparaît plus comme un dispositif normalisé, composé de séries d'objets graphiques mis en cohérence. Elle semble au contraire extraordinairement fragile, relevant d'un domaine d'activités dont l'utilité n'est pas clairement comprise et encore moins partagée. Bien que standardisé, le dispositif n'est pas stable en soi, il fait l'objet de nombreuses opérations qui visent à lui donner une place au sein d'une très grande entreprise qui comprend quarante-cinq mille salariés, dont l'immense majorité, à l'exception de ceux qui ont un poste qui lui est dédié ${ }^{3}$, a bien d'autres soucis que l'emplacement et la forme des panneaux installés en stations.

Une première manière de faire une place à la signalétique consiste à l'ériger en préoccupation au sein des activités de certaines personnes. C'est le cas par exemple pour les exploitants de station dont le métier est notamment de superviser l'état des espaces. Dans ce cadre, il leur est demandé de prêter une attention particulière à l'état des panneaux et de signaler des dégradations ou des absences, au même titre qu’ils signalent la présence

\footnotetext{
${ }^{3}$ À la RATP il y a avait au moment de notre enquête deux responsables de la normalisation et une dizaine de personnes chargées de l'installation et de la maintenance des panneaux.
} 
de graffiti sur les murs, celle de mauvaises odeurs, ou l'endommagement du mobilier. Cette obligation s'inscrit dans des formulaires standardisés et des processus organisationnels clairement identifiés (comme le circuit du signalement entre les stations et le service de maintenance), mais elle passe aussi par des échanges beaucoup plus anodins. II arrive par exemple qu'à l'occasion de leurs interventions, les agents de maintenance repèrent un élément de la signalétique manquant ou détérioré qui n'a pas été signalé. Ils cherchent alors à croiser l'exploitant de la station pour l'informer de la situation et en profitent pour insister sur le fait qu'elle représente un manquement au rôle d'exploitant, dont ils expliquent de manière plus ou moins explicite qu'il implique de se préoccuper de l'état des panneaux. D'une manière similaire, les deux responsables de la normalisation se trouvent régulièrement en position de rappeler les standards de la signalétique à un directeur de ligne ou un employé du service de communication en lui demandant de respecter une mise en page particulière ou d'utiliser telle couleur plutôt que telle autre.

Dans tous ces cas, il s'agit d'inscrire la signalétique en tant que dispositif stabilisé et normalisé à l'agenda de travailleurs qui ne sont pas enclin à la prendre en considération. Par l'intermédiaire de différents porte-parole, elle devient un objet clairement identifié et résistant, contraignant en partie les activités. Ces processus peuvent paraître anodins, ils sont pourtant essentiels et difficiles à réaliser. La signalétique est loin d'être une évidence. Pour illustrer cela, il suffit de prendre le cas des opérations de restauration des stations, initiées par le programme «Renouveau du Métro ». Dans ce cadre, des entreprises extérieures sont chargées de remettre à neuf les stations, y compris leurs modules de signalétique. Mais à l'occasion de la livraison des premières stations, de nombreux problèmes ont été rencontrés. Une station entièrement rénovée a même été considérée comme "livrée 》 alors qu'elle n'était dotée d'aucun panneau. Ni pour les responsables du chantier, ni pour ceux qui en avaient effectué la réception au sein de la RATP, cette situation n'avait suffi à empêcher l'ouverture de la station. La forme des modules avait beau être standardisée, leur nombre et leur emplacement précisément détaillés dans le cahier des charges de la station, cela n'a pas réussi à faire exister la signalétique. Depuis cet épisode, pour qu'elle " compte ", c'està-dire que sa présence et son absence face une différence (Cooren, 2004), on a ajouté aux documents normatifs et contractuels un représentant humain chargé de conditionner la validation du travail de rénovation des stations (et son paiement) à la présence effective, et conforme, des panneaux dans la station.

Le processus prend une tournure tout à fait particulière lorsque les porte-parole de la signalétique se trouvent confrontés à des domaines qu'ils considèrent comme concurrents. La consolidation de la signalétique s'apparente alors à une "lutte ", pour reprendre le terme des personnes concernées. II s'agit moins dans ce cas d'organiser la préoccupation pour les panneaux que de faire de leur présence même dans les espaces du métro un impératif qui ne peut être remis en cause à aucun prix.

Dans le cas de la RATP, la lutte la plus explicite est sans doute celle menée face à la régie publicitaire. Nous l'avons vu, la normalisation de la signalétique a opéré un ordonnancement des espaces, standardisant non seulement la forme et le contenu des modules graphiques, mais aussi leur emplacement. Au cours de ce processus, des places très précises ont été réservées pour les affiches publicitaires. De ce point de vue, la lutte est terminée et la cohabitation des deux formes graphiques est stabilisée. Mais la régie propose aussi régulièrement des événements et des campagnes expérimentales, dites " hors-média », qui constituent des menaces directes pour la signalétique aux yeux de ses représentants. La menace est d'autant plus forte que les arguments des uns et des autres sont très inégaux. Les supports publicitaires sont représentés par la régie qui associe à chaque mètre carré revendiqué un revenu financier, les porte-parole de la signalétique opposent à cela des principes dont l'efficience n'est pas traduite en chiffres, encore moins en retombées financières. La lutte prend des formes variées : elle peut consister à la présence en personne d'un représentant de la signalétique qui réagit à certains projets de la régie 
publicitaire, mais elle se joue aussi dans la rédaction de documents qui cherchent à définir les bonnes pratiques de la cohabitation. Lors de notre enquête, l'une des personnes du service de normalisation était ainsi chargée d'élaborer une "charte du bon usage de l'adhésivage dans les espaces voyageurs ", tant la pratique (qui consiste à recouvrir des portions de l'espace d'adhésifs publicitaires) était devenue répandue et jugée critique. Le but était clair : encadrer le plus possible, sans jugement de valeur, le processus de création et de commercialisation de ce type d'espaces publicitaires.

$\mathrm{Ce}$ dernier aspect montre à quel point l'aménagement des arenas, et notamment l'organisation des relations entre signes et espaces, peut devenir une question politique. Tout espace, surtout s'il est public, est le support potentiel d'une pluralité de formes graphiques. La signalétique n'est pas le seul dispositif susceptible de peupler les stations de métro. Sa place parmi d'autres "espèces d'écrits " ne va pas de soi et doit se gagner au prix d'une lutte permanente. Les travaux de sociologie urbaine qui ont fondé l'École de Chicago sont particulièrement utiles pour comprendre cette compétition (Hughes, 1936 ; Park, 1936). On peut en effet appréhender les espaces de transport comme des territoires à conquérir. Parce que leurs capacités d'exposition apparaissent limitées, ils sont le théâtre d'une compétition entre de nombreuses formes d'écrits qui prétendent l'investir4. De ce point de vue, la performativité des artefacts scripturaux doit donc être comprise non seulement comme le résultat d'une relation stabilisée entre signes et espaces, mais aussi comme un équilibre fragile au sein d'une certaine écologie graphique (Denis et Pontille, 2009, 2010a, cahp. 2).

Plus généralement, les processus décrits ici, qui se déroulent en coulisses, invitent à préciser l'idée selon laquelle la force des panneaux de la signalétique, leur capacité à faire faire, réside dans leur immuabilité. Ils montrent notamment qu'on ne peut pas considérer cette immuabilité comme une conséquence mécanique de la normalisation et de la standardisation. L'entrée dans l'activité des services de la RATP permet de comprendre que l'immuabilité est une qualité relative. II ne suffit pas que la signalétique soit détaillée en modules standardisés, dont le contenu, les couleurs et la forme sont répertoriés dans un document volumineux, pour que sa stabilité et son existence même soient assurées. II faut aussi trouver un moyen de tourner les regards des uns et des autres dans sa direction. La signalétique doit devenir un domaine de préoccupation, et donc un objet de travail, au sein des différents processus organisationnels déjà en place. En d'autres termes, si la performativité de ce type de dispositif peut s'appréhender via les formes spécifiques d'attention qu'il engage du côté des usagers (Denis et Pontille, 2010a, chap. 3), nous voyons ici qu'elle repose aussi sur une forme d'attention symétrique, qui se joue en coulisses. Pour que les modules de la signalétique deviennent effectivement fixes et immuables, ils doivent faire l'objet d'une certaine considération professionnelle, répartie sous différentes formes dans des lieux et des activités variés. Leur stabilité dépend d'une multitude de petites opérations dont l'agencement est un travail à part entière.

\section{Permanence du dispositif et instabilité des objets}

La mise en lumière des délicats assemblages sociotechniques sur lesquels repose quotidiennement l'immuabilité de la signalétique n'est qu'une première étape pour l'analyse de la performativité des objets graphiques. II faut aller jusqu'au bout des leçons que l'on peut en tirer. Nous l'avons vu, la sensibilisation des personnes à l'importance des modules de la signalétique n'est jamais faite une fois pour toutes. Ce qui compte, c'est au contraire la répétition au jour le jour des opérations de supervision, de réparation et de remplacement

\footnotetext{
${ }^{4}$ L'exemple le plus frappant dans cette perspective étant sans doute les graffitis. Dans leur cas, la lutte s'exprime d'une manière très différente de ce qui se passe avec la publicité. Elle ne s'opère pas seulement en coulisses, par l'intermédiaire de chartes et de documents normatifs, mais aussi directement dans les stations de métro, lieux essentiels (avec les tribunaux) de la confrontation; et les instruments qui équipent aussi bien leur installation que leur enlèvement soulèvent des enjeux très spécifiques, au premier rang desquels la dégradation matérielle.
} 
des éléments du dispositif graphique. Au delà de la seule distribution des tâches nécessaires à la félicité de tout le système, leur réitération incessante est elle-même un enjeu. L'immuabilité des modules de la signalétique et l'ordonnancement graphique des espaces qu'elle accomplit ne sont donc jamais acquis. Ils se rejouent à chaque instant, répartis dans les séquences de travail des exploitants de station, des ouvriers de maintenance, des responsables de la normalisation, et d'autres encore.

C'est une dimension centrale de l'idée même de maintenance. Maintenir, c'est assurer la stabilité des choses, les doter d'une permanence dont elles ne sont pas pourvues "naturellement ». Nous proposons de faire de ce travail de maintenance un point focal dans l'analyse de la performativité de l'écrit. C'est par lui que l'on peut dépasser l'aporie que nous évoquions en introduction à propos des l'analyse des pratiques d'écriture, et refuser de faire de l'immuabilité des artefacts graphiques un principe explicatif.

Ce déplacement théorique consiste à revenir, du point de vue des objets, aux principes de l'ethnométhodologie tels que les a exposés H. Garfinkel (1967) et à la théorie de l'ordre social développée par E. Goffman (1991). Pour résumer, ces deux auteurs insistent sur la remise en jeu nécessaire des fondamentaux de l'ordre social dans les relations de face-àface. L'ordre des échanges est toujours un ordre accompli en situation. Pour le dire autrement, il existe très peu de choses dans notre monde qui soient identifiées, instaurées, une fois pour toutes, c'est-à-dire sur lesquelles nous serions tous d'accord si nous avions à les définir en détails. F. Cooren (2010) développe ces propositions en montrant qu'aucun principe, aucune règle d'organisation des relations sociales ne peut se passer de formes d'incarnation répétées. II faut toujours les donner à voir, les faire exister, à « chaque nouvelle première fois » (" each another next first time » Garfinkel, 2002, p. 98).

Nous l'avons vu, le geste essentiel de B. Latour consiste à ajouter à ces perspectives théoriques un intérêt pour les dynamiques de stabilisation et le rôle crucial qu'y tiennent les artefacts. Mais l'observation des pratiques quotidiennes dans les services de normalisation et de maintenance de la signalétique montre qu'il ne faut pas tomber dans l'excès inverse. Ce serait faire preuve d'un matérialisme borné que de considérer la stabilité comme un corollaire de tout artefact. Les propriétés matérielles ne sont pas intrinsèques aux objets, elles s'actualisent au jour le jour. Comme le montrent C. Henke (2000), et S. Graham et N. Thrift (2007), il est ainsi particulièrement riche d'adopter sur les objets la même perspective que Garfinkel et Goffman adoptent sur la vulnérabilité des échanges ordinaires. L'ordre des choses, tout comme l'ordre des interactions, s'entretient et se répare.

Adopter cette perspective ne revient pas à dire que les objets, en l'occurrence les objets écrits, ne sont pas stables et ne permettent pas d'ordonnancer l'environnement. Cela permet en revanche d'étudier plus précisément le caractère relatif de leur immuabilité. Non seulement, le dispositif de la signalétique fait l'objet de sensibilisations constantes auprès de nombreuses personnes de l'entreprise de transport, mais les modules eux-mêmes ne sont pas stables pour tout le monde, tout le temps. Leur stabilité est une stabilité exposée. Elle est adressée aux voyageurs, tournée vers eux. Nous pensons que la compréhension des formes de performativité des artefacts passe par la prise en compte de cette dimension des objets et dans le refus de se focaliser sur les seules dynamiques aval, que ce soit en termes d'« usages » ou d'« effets ». Tout comme l'identité pour E. Goffman (1973) est toujours une identité projetée, une performance qui n'est possible que parce qu'elle repose sur des activités de coulisses, l'identité des objets, leur immuabilité, doit pouvoir être observée comme le résultat fragile d'un travail constant de maintenance.

Mais de quoi ce travail est-il composé exactement, que nous apprend-il ? Les enquêtes sociologiques dans le domaine se focalisent généralement sur les personnes. Elles visent à mettre en lumière le continent des activités qui composent au jour le jour l'infrastructure du monde. Dans ce cas, le chercheur se donne pour mission de faire remonter à la surface le travail invisible afin de redécouvrir " the mess obscured by the boring sameness of the 
information represented » (Star, 1999, p. 385). Ce mouvement est proche de celui que font la sociologie du travail et l'ergonomie en cherchant à mettre en lumière la richesse et la complexité du « travail réel » face au «travail prescrit ». II insiste sur les compétences des travailleurs invisibles, notamment leurs capacités à improviser et à inventer des solutions in situ, essentielles à la réussite du travail de réparation et de maintenance (Henke, 2000, p. 66-69).

Mais l'étude du travail de maintenance est aussi précieuse pour l'analyse des objets euxmêmes. Suivre les exploitants de station ou les ouvriers de la maintenance, c'est étudier des lieux et des moments où les modules de la signalétique ne sont plus sous le joug de l'évidence. C'est explorer d'autres modes d'existence par lesquels ils ne sont plus, dans les termes heideggerriens, " ready-to-hand ", c'est-à-dire transparents à l'usage, mais "present-at-hand ", c'est-à-dire considérés séparément de leur utilisation ordinaire, ce qui arrive lorsque les artefacts se cassent ou tombent en panne (Verbeek, 2004).

Cela change tout dans la compréhension que l'on peut avoir de la performativité des écrits exposés. II ne faut pas porter le regard sur les seules stabilité et durabilité des panneaux de la signalétique puisque, aux yeux des personnes chargées de leur surveillance et de leur réparation, ils ne sont jamais tout à fait stables ${ }^{5}$. C'est même cette sensibilité à l'instabilité des panneaux, aux changements dont ils font preuve, qui est au cœur de leur travail. Elle constitue leur expertise. C'est précisément l'enjeu des processus que nous avons décrits plus haut : ils nourrissent un travail constant qui vise à faire porter l'attention de nombreuses personnes sur les mutations qui ont cours au sein du dispositif graphique. On apprend, par exemple, à un exploitant de station qu'un panneau qui a été rayé à la clef pour produire un " scratchiti » n'est plus vraiment un panneau, en tout cas qu'il n'est plus un panneau de la signalétique et qu'il faut donc le remplacer pour assurer l'immuabilité du système. La maintenance se met en œuvre lorsque des personnes développent des capacités à percevoir les différences à l'intérieur d'un système d'objets qui peut paraître stable au premier regard et dans l'environnement hybride que la signalétique compose ; lorsqu'elles deviennent attentives à l'usure, et qu'elles savent percevoir les traces infimes de l'absence, leur permettant de juger qu'un couloir n'est plus véritablement un couloir si l'un de « ses » panneaux vient à manquer.

La maintenance consiste donc à développer une certaine forme d'expertise qui permet de percevoir des seuils de variabilité au service d'une immuabilité projetée vers des usagers. Autrement dit, si le dispositif de la signalétique est un assemblage d'objets immuables du point de vue des voyageurs, c'est parce qu'il est inversement fait de variations et de mutations aux yeux de tous ceux qui se chargent de sa maintenance. On peut faire la même réflexion à propos de l'immobilité. La fixité des panneaux, leur permanence dans les espaces du métro, est liée à leur mobilité aux yeux et dans les mains des mainteneurs. Ils doivent pouvoir les décrocher et les remplacer et, pour cela, les faire circuler des lieux de leur conception et de leur fabrication jusqu'à leur emplacement en station.

En soulignant l'importance des activités de maintenance, il ne s'agit donc pas de défendre un point de vue qui serait par principe définitivement opposé au vocabulaire de l'immuabilité. C'est l'enfermement dans ce seul registre qui représente un problème. Comme l'ont montré plusieurs travaux à propos de la notion de réseau sociotechnique développée par Callon et Latour, il faut se méfier des ontologies que l'on plaque en tant que chercheur sur les objets que l'on étudie (Mol et Law, 1994 ; de Laet et Mol, 2000 ; Law, 2002). Prenant le cas d'une innovation technologique (le terrain favori de la théorie de l'acteur-réseau), $M$. de Laet et $A$. Mol (2000) montrent par exemple que le succès de la pompe du désert au Zimbabwe ne repose pas sur les opérations qui en font une boîte noire aux formes et aux fonctions stabilisées. C'est au contraire parce qu'elle est souple au changement que la pompe du

\footnotetext{
${ }^{5}$ Réinjecter une dose de stabilité forcée au nom de la dimension graphique des objets reviendrait à tomber dans ce que S.L. Star appelle très justement un « formalisme naïf » (Star, 1995, p. 16).
} 
désert et ses usages se sont aussi bien développés. Les auteures en concluent, relayées par J. Law (2002) dans une discussion détaillée, qu'il faut se défaire d'une vision exclusivement nourrie de la notion de réseau pour élargir la gamme des ontologies étudiées. C'est un mouvement identique que nous proposons ici. Prendre en compte le travail généralement invisible qui est effectué pour maintenir un dispositif tel que la signalétique permet d'ouvrir l'analyse des artefacts à la variété et à la relativité de leurs ontologies. Les panneaux sont immuables et muables, mobiles et immobiles d'une situation à l'autre ${ }^{6}$.

Notre détour forcé par la maintenance montre qu'on ne peut pas répondre à la question de la performativité des artefacts graphiques par un catalogue de «propriétés " plus ou moins intrinsèques aux écrits exposés. Nous voyons au contraire que cette performativité repose sur la possibilité d'un jeu entre ces propriétés. C'est le cœur du travail de maintenance : une série d'opérations très concrètes, qui consistent à assurer, au jour le jour, une stabilité et une permanence à des objets graphiques, en mettant en œuvre les conditions de reconnaissance de leurs transformations incessantes.

\section{Conclusions : conditions de félicité et mode d'existence des écrits exposés}

Les écrits exposés sont performatifs en tant qu'ils participent de l'ordonnancement du monde. La présence de certains artefacts graphiques dans les lieux publics transforme un espace en un environnement hybride où se mêlent étroitement dimensions géographiques, architecturales et langagières. En éclairant les coulisses de cette présence, nous avons montré que cette performation n'avait rien de mécanique, mais qu'elle reposait sur un travail constant de maintenance. Une telle démarche permet de souligner la complexité, et la fragilité, des processus d'aménagement de ce que J. Lave (1998) appelle les arenas c'est-à-dire les espaces matériels et sémiotiques qui sont mis à la disposition des usagers. Aux multiples ajustements que font les personnes qui mobilisent leur environnement spatial et les artefacts qui s'y trouvent pour produire un cadre d'action pertinent (un setting), correspond une série d'opérations délicates de stabilisation et d'entretien.

D'un certain point de vue, notre analyse a donc consisté à interroger la face habituellement cachée des «conditions de félicité » (Austin, 1970) de la signalétique en tant que dispositif d'écrits exposés. Mais la mise en lumière du travail de maintenance met finalement à mal l'idée même de conditions de félicité. Elle en redéfinit en tout cas les contours. S'il y a dans la notion proposée par Austin le refus de faire de la performativité une propriété des seuls énoncés, les conditions de félicité restent, dans son modèle, relativement inertes. Elles s'apparentent à des procédures et des principes conventionnels stabilisés qu'il suffirait de respecter pour réussir. Cette position a été critiquée par P. Bourdieu (1982), mais uniquement pour défendre l'importance de structures sociales et de jeux de pouvoir préexistants. Elle fait depuis quelques années l'objet d'un renouvellement dans de nombreuses disciplines à travers des travaux qui décrivent les conditions de félicité en tant que situations concrètes d'accomplissement des énonciations performatives (Denis, 2006). Notre analyse du travail de maintenance s'inscrit dans le même mouvement. La prise en considération des activités répétées qui sont nécessaires au maintien de la stabilité des écrits exposés donne à voir le caractère irrémédiablement dynamique de leurs conditions de félicité. Celles-ci ne sont pas isolables dans une liste de caractéristiques à rassembler : on ne peut les repérer qu'en tant qu'opérations variées accomplies jour après jour.

\footnotetext{
6 II ne faut toutefois pas réduire la variabilité des ontologies aux passages de la frontière invisible entre usagers et coulisses. Si l'on suit les ouvriers qui installent les modules de la signalétique dans leurs activités quotidiennes, on voit comment les panneaux passent régulièrement d'une ontologie à une autre au fil de l'action (Denis et Pontille, 2010b).
} 
De manière plus générale, que peut nous apprendre cette analyse de la signalétique sur la performativité des artefacts graphiques ? Nous l'avons vu, les panneaux du métro sont des " écritures exposées » (Petrucci, 1993), en ce sens, ils soulèvent des questions centrales quant au rôle de la spatialité dans leur force performative (Fraenkel, 2006). Mais l'importance du travail de maintenance dont ils font l'objet montre que la dimension temporelle y tient aussi une place particulièrement sensible. C'est sur ce point que la comparaison avec d'autres types d'objets graphiques nous semble pertinente.

Tout au long de ses conférences, J.-L. Austin (1970) enracine, à travers une série d'exemples bien connus (le baptême, le mariage, la promesse), la définition de la performativité dans une instantanéité qui permet la mise en contraste d'un "avant » et d'un « après ». Au même titre que les travaux sur les écrits juridiques (Fraenkel, 2006 ; Pontille, 2006), l'analyse des écrits exposés insiste au contraire sur la capacité des énoncés performatifs à durer et invite donc à sortir l'étude de la performativité de son modèle canonique, restreint à l'ici et maintenant de l'énonciation. B. Fraenkel (2006) a développé cette réflexion à propos du droit en mettant en lumière la « performativité latente » des actes juridiques. Ceux-ci ne sont pas performatifs en eux-mêmes : pour que leur garantie opère, il faut pouvoir réactiver leur force. Leur vigueur est réveillée par des activités de lecture et d'écriture spécifiques. Pour être convoquées (c'est le cas par exemple du testament), les pièces sont manipulées, lues à haute voix, en partie recopiées, etc. La performativité des écrits juridiques est latente en ce sens qu'elle est décomposée en moments plus ou moins éloignés.

Si la performativité des écrits exposés est aussi une question de durée, elle diffère sensiblement de celle des écrits juridiques. Les conditions de leur durabilité, c'est-à-dire de leur pérennité, mettent en lumière deux modes d'existence de l'écrit. En dehors des moments où ils sont réactivés, la force des écrits du droit est assurée par l'efficacité de leur archivage (Pontille, 2006). Pour qu'ils soient toujours en vigueur, les actes juridiques doivent être entreposés à l'abri de toute dégradation possible. Les écrits exposés, comme leur nom l'indique, ne peuvent pas bénéficier de ce traitement. Leur force ne réside pas dans leur capacité à être remobilisés à des moments précis, mais dans leur disponibilité constante.

Dans les deux cas, la question de la durabilité des énoncés se traduit différemment : à la vigueur des uns répond la vivacité des autres. Les écrits juridiques relèvent d'une performativité latente qui repose sur un régime de conservation, tandis que les écrits exposés relèvent d'une performativité permanente qui repose sur un régime de maintenance.

La comparaison des deux modes d'existence de l'écrit permet de préciser encore en quoi consiste le travail de maintenance. La performativité latente du droit n'exclut pas certaines formes de réparation et d'intervention sur les écrits eux-mêmes. Mais celles-ci ont cette particularité de devoir être visibles (par l'ajout de modifications, de biffures...) pour être valables (Pontille, 2009). Dans le cas de la signalétique, les opérations de maintenance restent invisibles. Leur but est de restaurer le dispositif dans son ensemble, en rendant au panneau concerné son aspect initial. C'est une dimension centrale des écrits exposés : leur capacité à se donner " seuls ", toujours identiques, à la disposition du public. Cela implique une certaine écologie du visible et de l'invisible (Star et Strauss, 1999). La performativité de la signalétique semble reposer sur la mise en invisibilité des agencements fragiles qui la rendent possible et du travail incessant qu'elle nécessite. Les écrits exposés ne performent donc pas seulement un environnement hybride tourné vers le public. En donnant forme au décor, ils donnent aussi forme à ses coulisses. Leur félicité tient autant dans la visibilité du premier que dans l'invisibilité des secondes. 


\section{Remerciements}

Nous remercions vivement les évaluateurs de la revue Réseaux pour leurs commentaires et suggestions qui nous ont permis d'expliciter divers aspects de ce texte. Cet article s'appuie sur une recherche menée dans le cadre d'un programme intitulé «Écologies et Politiques de l'Écrit " (sous la responsabilité de C. Licoppe et de B. Fraenkel) et financé par l'Agence Nationale de la Recherche (ANR-05-BLAN-0272-02).

\section{Références}

AMIN A. et THRIFT N. (2002), Cities. ReimagiEEg the urbaE, Cambridge, Blackwell.

ARTIÈRES P. et RODAK P. (2008), «Écriture et soulèvement. Résistances graphiques pendant l'état de gueree en Pologne (13 décembre 1981 - 13 décembre 1985) ", GeEèses, n70, p. 120-139.

AUSTIN J.L. (1970), QuaEd dire c'est faire, Paris, Le Seuil.

BIJKER W.E. et LAW J. (1992), ShapiEg TechEology / BuildiEg Society, Cambridge, MIT.

BOURDIEU P. (1982), Ce que parler veut dire. L'écoEomie des échaEges liEguistiques, Paris, Fayard.

CALLON M., LAW J., RIP A. (1986), MappiEg The DyEamics of ScieEce aEd TechEology: Sociology of ScieEce iE the Real World, London, Macmillan.

COOREN F. (2004), "Textual Agency: How Texts Do Things in Organizational Settings ", OrgaEizatioE, vol. 11(3), p. 373-393.

COOREN F. (2010), ActioE aEd AgeEcy iE Dialogue: PassioE, IEcarEatioE aEd VeEtriloquism, Amsterdam / Philadelphia, John Benjamins.

DE LAET M. et MOL A. (2000), «The Zimbabwe Bush Pump: Mechanics of a Fluid Technology », Social Studies of ScieEce, vol. 30(2), p. 225-263.

DENIS J. (2006), « Les nouveaux visages de la performativité », Études de commuEicatioE, n²9, p. 7-24.

DENIS J. (2009a), « Le travail invisible de l'information », in C. Licoppe (dir.) L'évolutioE des cultures Eumériques, de la mutatioE du lieE social à l'orgaEisatioE du travail, Paris, FYP, p. 117-123.

DENIS J. (2009b), “ Sécurité informatique et valeur des écrits au travail », SemeE n² 28, p. 85-100.

DENIS J. et PONTILLE D. (2009), " L'écologie informationnelle des lieux publics. Le cas de la signalétique du métro ", in C. Licoppe (dir.) L'évolutioE des cultures Eumériques, de la mutatioE du lieE social à l'orgaEssatioE du travail, Paris, FYP, p. 94-101.

DENIS J. et PONTILLE D. (2010a), Petite sociologie de la sigEalétique. Les coulisses des paEEeaux du métro, Paris, Les presses des Mines.

DENIS J. et PONTILLE D. (2010b), «Placing Subway Signs. Practical Properties of Signs at Work », Visual CommuEicatioE, (à paraître).

DENIS J. et PONTILLE D. (2010c), «The Graphical Performation of a Public Space. The Subway Signs and their Scripts », in C. Coletta, F. Gabbi et G. Sonda (dir.) UrbaE Plots, OrgaEiziEg Cities, Farnham, Ashgate, (à paraître).

EISENSTEIN E. (1991), La révolutioE de l'imprimé daEs l'Europe des premiers temps moderEes, Paris, La découverte.

FRAENKEL B. (2002), Les écrits de New York. Septembre 2001, Paris, Textuel.

FRAENKEL B. (2006), «Actes écrits, actes oraux : la performativité à l'épreuve de l'écriture ", Études de commuElcatioE, $\mathrm{n}^{\circ} 29$, p. 69-93.

FRAENKEL B. (2007), “ Actes d'écriture : quand écrire c'est faire », LaEgage et Société, n 121-122, p. $101-112$.

FULLER G. (2002), «The Arrow-Directional Semiotics: Wayfinding in Transit », Social Semiotics, vol. 12(3), p. 231-178.

GARFINKEL H. (1967), Studies iE EthEomethodology, Englewood-cliffs, Prentice-Hall. 
GARFINKEL H. (2002), Ethnomethodology's Program. Working Out Durkheim's Aphorism, New York, Rowman and Littlefield Publishers.

GOFFMAN E. (1973), La Mise en scène de la vie quotidienne. Tome 1: la présentation de soi, Paris, Ed. de Minuit.

GOFFMAN E. (1991), Les Cadres de l'expérience, Paris, Ed. de Minuit.

GOODY J. (1979), La Raison graphique, Paris, Ed. de Minuit.

GRAHAM S. et THRIFT N. (2007), "Out of Order. Understanding Repair and Maintenance ", Theory, Culture \& Society, vol. 24(3), p. 1-25.

GROSJEAN M. et LACOSTE M. (1998), «L'oral et l'écrit dans les communications de travail ou les illusions du "tout écrit" », Sociologie du travail, vol. XL(4), p. 439-465.

HENKE C. R. (2000), «The Mechanics of Workplace Order: Toward a Sociology of Repair », Berkeley Journal of Sociology, $n^{\circ} 44$, p. 55-81.

HUGHES E. C. (1936), "The Ecological Aspect of Institutions », American Sociological Review, vol. 1 (2), p. 180-189.

HUTCHINS E. (1995), Cognition in the Wild. Cambridge, MIT Press.

KÄRRHOLM M. (2007), "The Materiality of Territorial Production: A Conceptual Discussion of Territoriality, Materiality, and the Everyday Life of Public Space ", Space and Culture, vol. 10(4), p. 437-453.

LASH S. et URRY J. (1994), Economies of Signs and Space, London, Sage.

LATOUR B. (1985), "Les «Vues » de l'esprit. Une introduction à l'anthropologie des sciences et des techniques », Culture Technique, $n^{\circ} 14$, p. 4-29.

LATOUR B. (1993), “ Le topofil de Boa-Vista. La référence scientifique : montage photophilosophique ", in B. Conein, N. Dodier et L. Thévenot (dir.) Les objets dans l'action. De la maison au laboratoire, Paris, Éditions de l'EHESS, p. 187-216.

LATOUR B. et WOOLGAR S. (1988), La vie de laboratoire, Paris, La Découverte.

LATOUR B. et HERMANT É. (1998), Paris ville invisible, Paris, Les empêcheurs de penser en rond / La Découverte.

LAVE J. (1988), Cognition in Practice. Mind, Mathematics and Culture in Everyday Life, Cambridge, Cambridge University Press.

LAW J. (2002), « Objects and Spaces », Theory, Culture \& Society, vol. 19(5-6), p. 91-105.

LÉVY E. (2001), “Saisir l'accessibilité, les trajets voyageurs à la gare du Nord », in M. Grosjean et J.P. Thibaud (dir.), L'espaceurbainenméthodes, Paris, Parenthèse, p. 47-62.

MOL A. et LAW J. (1994), "Regions, Networks and Fluids: Anaemia and Social Topology », Social Studies of Science, vol. 24(4), p. 641-671.

PARK R. E. (1936), « Human Ecology », American Journal of Sociology, vol. 42(1), p. 1-15.

PETRUCCI A. (1993), Jeux de lettres. Formes et usages de linscription en Italie 11e-20e siècles, Paris, Éditions de l'EHESS.

PONTILLE D. (2006), « Produire des actes juridiques », in A. Bidet, A. Borzeix, T. Pillon, G. Rot et F. Vatin (dir.) Sociologie du travail et activité, Toulouse, Octares, p. 113-126.

PONTILLE D. (2009), "Écriture et action juridique. Portrait de l'huissier de justice en réparateur », Semen, 28, p. 15-31.

RELIEU M. (1999), “ Travaux publics. La dynamique d'une situation problématique », in (dir.) La logique des situations. Nouveaux regards sur l'écologie des activités sociales, Paris, Éditions de I'EHESS, p. 95-117.

RELIEU M. et TERZI C. (2004), " Les politiques ordinaires de la vie urbaine. L'organisation de l'expérience publique de la ville ", in D. Pasquier et D. Cefaï (dir.) Les sens du public. Publics politiques, publics médiatiques, Paris, PUF, p. 373-397. 
SHARROCK W.W. et ANDERSON D.C. (1979), "Directional Hospital Signs as Sociological Data », Information Design Journal,, vol. 1(2), p. 81-94.

SCOLLON R. et SCOLLON S.W. (2003), Discourses in Place. Language in the Material World, London, Routledge.

SIMMEL G. ([1909] 1994), «Bridge and Door », Theory Culture \& Society, vol. 11(5), p. 5-10.

STAR S.L. (dir.) (1995), The Ecologies of Knowledge: Work and Politics in Science and Technology, New York, State University of New York Press.

STAR S.L. (1999), «The Ethnography of Infrasctructure ", American Behavioural Scientist, vol. 43(3), p. 377-391.

STAR S.L. et STRAUSS A. (1999), "Layers of Silence, Arenas of Voice: The Ecology of Visible and Invisible Work », Computer Supported Cooperative Work, n 8, p. 9-30.

SUCHMAN L. (1987), Plans and Situated Actions, New York, Cambridge University Press.

TIMPF S. (2002), "Ontologies of Wayfinding: a Traveler's Perspective ", Networks and Spatial Economics, vol. 2(1), p. 9-33.

VERBEEK P. (2004), What Things Do: Philosophical Investigations on Technology, Agency and Design, University Park, Pennsylvannia State University Press. 